\title{
Detection of human neutrophil elastase (HNE) on wound dressings as marker of inflammation
}

\author{
Ana V. Ferreira ${ }^{1}$ - Ilana Perelshtein ${ }^{2} \cdot$ Nina Perkas $^{2} \cdot$ Aharon Gedanken $^{2}$. \\ Joana Cunha $^{1} \cdot$ Artur Cavaco-Paulo $^{1}$ (i)
}

Received: 3 August 2016/Revised: 12 September 2016/Accepted: 23 September 2016/Published online: 15 October 2016

(C) Springer-Verlag Berlin Heidelberg 2016

\begin{abstract}
Chronic wound fluids have elevated concentration of human neutrophil elastase (HNE) which can be used as inflammation/infection marker. Our goal is to develop functional materials for fast diagnosis of wound inflammation/ infection by using HNE as a specific marker. For that, fluorogenic peptides with a HNE-specific cleavage sequence were incorporated into traditional textile dressings, to allow real-time detection of the wound status. Two different fluorogenic approaches were studied in terms of intensity of the signal generated upon HNE addition: a fluorophore 7amino-4-trifluormethylcoumarin (AFC) conjugated to a HNE-specific peptide and two fluorophore/quencher pairs (FAM/Dabcyl and EDANS/Dabcyl) coupled to a similar peptide as a Förster resonance energy transfer (FRET) strategy. Also, two immobilization methods were tested: sonochemistry immobilization onto a cotton bandage and glutaraldehyde (GTA)-assisted chemical crosslinking onto a polyamide dressing. The immobilized fluorogenic AFC peptide showed an intense fluorescence emission in the presence of HNE. HNE also induced an enhanced fluorescent signal with the EDANS/Dabcyl FRET peptide which showed to be a more sensitive and effective strategy than the AFC peptide. However, its chemical immobilization onto the polyamide
\end{abstract}

Electronic supplementary material The online version of this article (doi:10.1007/s00253-016-7889-6) contains supplementary material, which is available to authorized users.

Artur Cavaco-Paulo

artur@deb.uminho.pt

1 Centre of Biological Engineering (CEB), University of Minho, 4710-057 Braga, Portugal

2 Department of Chemistry, Bar-Ilan Institute of Nanotechnology and Advanced Materials, Bar-Ilan University,

IL-52900 Ramat-Gan, Israel dressing greatly decreased its detection, mainly due to the more difficult access of the enzyme to the cleavage sequence of the immobilized peptide. After optimization of the in situ immobilization, it will be possible to use these fluorescencefunctionalized dressings for an effective and specific monitoring of chronic wounds by simply using a portable ultraviolet (UV) light source. We envision that the development of this point-of-care medical device for wound control will have a great impact on patient's life quality and reduction of costs on health care system.

Keywords Human neutrophil elastase (HNE) · Förster resonance energy transfer (FRET) · Fluorogenic detection . Functionalized wound dressing $\cdot$ Chronic wounds

\section{Introduction}

Human neutrophil elastase (HNE) is one of the most abundant neutral proteinases present in chronic wounds (Weitz et al. 1986; Kolaczkowska and Kubes 2013), being significantly active in pressure and diabetic ulcers (Weiss et al. 1986; Herrick et al. 1992a; Korkmaz et al. 2008a). Recent studies have demonstrated that HNE activity is elevated in fluids from chronic wounds $(22.97 \pm 13.27 \mathrm{U} / \mathrm{mL})$ and reduced in healing wounds $(2.89 \pm 1.27 \mathrm{U} / \mathrm{mL})$ (Vasconcelos et al. 2010; Hasmann et al. 2011; Vasconcelos et al. 2011; Barros et al. 2013). According to this, we intend to use HNE activity as a wound inflammation/infection marker by using fluorogenic peptide sensors with HNE-specific cleavage sequence embedded into a dressing material (as schematized in Fig. 1). Briefly, HNE enzyme present in the chronic wound fluid will recognize and cleave the sensor peptides, leading to visually detectable modification on the dressing, thus discriminating the wound status. 


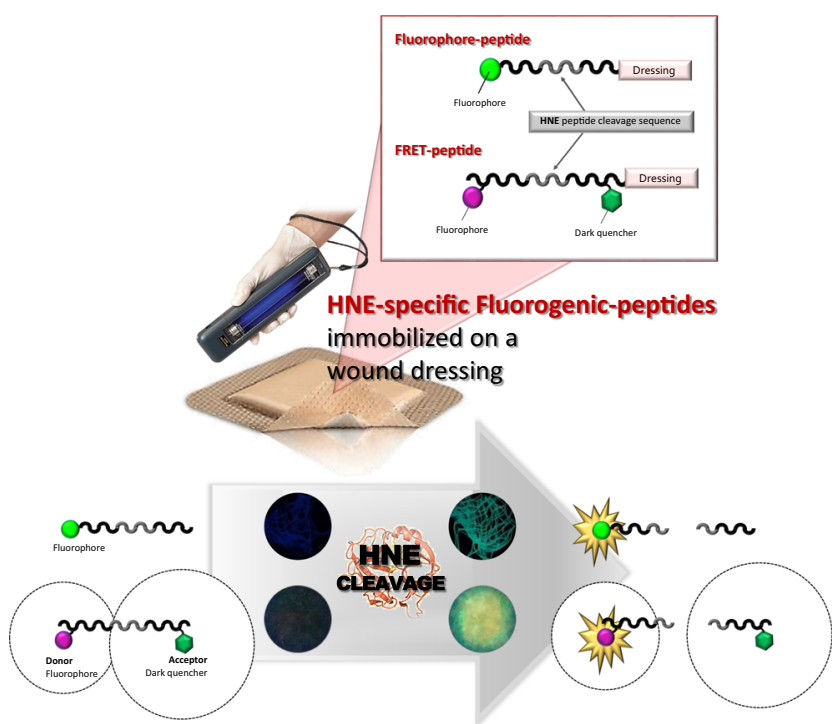

Fig. 1 Embedding of fluorogenic peptide sensors with HNE-specific cleavage sequence into a wound dressing material. HNE enzyme present in the chronic wound fluid will recognize and cleave the sensor peptides, leading to visually detectable modification on the dressing, thus discriminating the wound status

Nowadays, fluorescence systems are widely used in many applications in biotechnology fields, being a method of choice for biological and medical diagnosis due to its inherent sensitivity, selectivity, ease of implementation, response time, spatial resolution and also growing availability of commercial products (Lakowicz 2006; Adhikari et al. 2014). A possible limitation for the use of fluorogenic substrates is its direct visual assessment, since the human eye is not a great spectral detector and cannot easily detect fluorescence changes when white light is used as excitation source. To overcome this, we aim to develop a simple method for visual assessment of HNE in wound dressings by using a portable ultraviolet (UV) light source (commonly known as black light) to excite the fluorophores and allow the detection of the enzyme's activity on those fluorescent substrates.

In this work, to evaluate HNE activity, we firstly used a commercially available fluorogenic HNE-specific peptide, $\mathrm{N}$-(methoxysuccinyl)-alanine-alanine-proline-valine-7-amino-4-trifluormethylcoumarin (MeOSuc-AAPV-AFC), which releases AFC after peptidolysis, leading to the generation of a fluorescent signal. In a second approach, we designed a short peptide comprising a specific HNE cleavage sequence coupled to a fluorescence quenching system based on Förster resonance energy transfer (FRET). As described in literature, the quencher molecule absorbs the light emitted by fluorophore molecule, being this interaction distancedependent (Carmona et al. 2009; Fan et al. 2012). Hence, the quencher and fluorophore molecules were paired at opposite terminals of the HNE-specific peptide sequence. Moreover, the quencher molecule chosen is a dark quencher, meaning that the energy absorbed from the fluorophore is released in the form of heat instead of light (Lakowicz 2006). Therefore, it is not necessary to discriminate the wavelength of the light emitted by the system, since there will only be fluorescence emission when the peptide is cleaved and fluorophore and quencher are not in spatial vicinity.

A variety of immobilization techniques and approaches can be used to incorporate the fluorogenic sensors onto the wound dressing matrix. Sonochemical immobilization method is one of the most used and effective coating methods for textiles (Gedanken 2004; Perkas et al. 2007). Previously, we published several works showing that the deposition of a large variety of nanoparticles (NPs) on different materials by using the sonochemical method can be successfully achieved (Pol et al. 2003; Perkas et al. 2007; Perelshtein et al. 2009). In general terms, when high-intensity ultrasound is applied to the peptide aqueous solution, the peptide molecules are adsorbed on the formed acoustic bubbles (Sunartio et al. 2007). When the cavity collapses, the absorbed molecules are exposed to extreme localized conditions of temperature and pressure. As a consequence, these molecules impact on each other and NPs of the organic compound are produced. In our case, the cavitation occurs not only in liquid but also in a solid-liquid interface, i.e. near textile surface, due to increased nucleation rate in the presence of a solid material (Mersmann 2001). After the bubbles collapse, high-speed jets of the liquid throw the generated peptide NPs at high speed towards the textile cotton surface where they remained embedded (Perelshtein et al. 2009; Perelshtein et al. 2015). To achieve a stronger and stable immobilization, covalent binding through chemical reaction is another option (Brena et al. 2013). This chemical immobilization process involves two main steps: activation of the support matrixes (in this case, the textile dressing) and coupling of the interest compound (the sensor) to the activated matrices (Trevan 1988; Brena et al. 2013). The covalent bonds are normally formed through amide, ether, thioether or carbamate bonds (Trevan 1988; Brena et al. 2013).

Either through a sonochemical or chemical immobilization method, the embedding of the developed fluorogenic HNEspecific sensors into a functionalized wound dressing material will allow in situ and real-time assessment of the wound status at an early stage of inflammation/infection. In contrast to other off-site methods, this novel approach would provide a fast and objective diagnosis, without the need of expensive and longlasting analytic methods (Harding et al. 2011).

\section{Materials and methods}

Materials All chemicals were analytical graded and purchased from Sigma-Aldrich, namely glutaraldehyde (GTA) $50 \%$ solution in water, sodium hydroxide $(\mathrm{NaOH})$, hydrochloric acid $(\mathrm{HCl})$, pararosaniline hydrochloride (PS) dye, the enzyme HNE and synthetic HNE substrate pyroglutamate- 
proline-valine-p-nitroaniline (Glp-PV-pNA). The fluorogenic HNE substrate $N$-(methoxysuccinyl)-alanine-alanine-proline-valine-7-amino-4-trifluormethylcoumarin (MeOSuc-AAPV-AFC) was purchased from Abcam, and FRET peptides H-(FAM)lysine-alanine-alanine-proline-valine-lysine(Dabcyl)glycine-OH (FAM-AAPV-Dabcyl) and H-(EDANS)glutaminealanine-alanine-proline-valine-lysine(Dabcyl)-glycine-OH (EDANS-AAPV-Dabcyl) were synthetized by JPT Peptide Technologies GmbH (Germany). The respective molecular weight and structure of the chromogenic and fluorogenic HNE substrates are summarized in Table 1. Commercial cotton gauze with $17 \mathrm{~g} / \mathrm{m}^{2}$ was used for sonochemical coating. Commercial polyamide 6.6 fabric (nylon), a plain woven structure with $63 \mathrm{~g} /$ $\mathrm{m}^{2}$, was supplied by Rhodia (Switzerland).

Instrumentation High-intensity ultrasonic horn, Ti horn, $20 \mathrm{kHz}, 750 \mathrm{~W}$ at $30 \%$ efficiency, was used for sonochemical coating reactions. The morphology of sonochemically coated cotton was studied by high-resolution scanning electron microscope (HRSEM), by using a Magellan FEI, at an accelerating voltage over the range of $5-15 \mathrm{kV}$. All absorbance and fluorescence measurements were carried out in 96-well microplates by using a Synergy Mx reader (Bio-Tek Instruments, Inc., USA) spectrometer and Gen5 ${ }^{\mathrm{TM}}$ Data Analysis Software. Also, a fluorescence chamber equipped with UV light of $366 \mathrm{~nm}$ was used for visual assessment of the FRET system.

\section{Methods}

\section{HNE activity assessment}

HNE activity was evaluated with the synthetic substrates in accordance with the protocol supplied by Sigma-Aldrich. Briefly, the standard HNE activity assay consisted in mixing the enzyme $(0.5 \mathrm{U} / \mathrm{mL})$ with $25-1500 \mu \mathrm{M}$ of substrate, in a final volume of $300 \mu \mathrm{L}$ of reaction buffer (0.1 M HEPES + $0.5 \mathrm{M} \mathrm{NaCl}, \mathrm{pH}$ 7.5). A blank consisting only in buffer and substrate was also included in these assays. The kinetic constants were determined from the initial rates of hydrolysis by the Lineweaver-Burk method. All enzymatic assays were conducted at $37^{\circ} \mathrm{C}$ for $10 \mathrm{~min}$ with triplicates, and the absorbance was measured at $405 \mathrm{~nm}$ by UV-Vis spectrophotometry.

\section{Characterization of synthetic and commercially available HNE substrates and sonochemical immobilization on wound dressings}

Commercially available elastase substrates MeOSuc-AAPV$\mathrm{AFC}$ and Glp-PV-pNA were dissolved in water at a final concentration of $0.1 \mathrm{~g} / \mathrm{L}$, corresponding to a molar concentration of 147 and $225 \mu \mathrm{M}$, respectively. Then, the substrates were deposited onto a previously washed cotton bandages $\left(7 \times 7 \mathrm{~cm}^{2}\right)$ for $5 \mathrm{~min}$ by using a high-intensity ultrasonic horn. The sonication was carried out in a thermo-stated bath at $16^{\circ} \mathrm{C}$. The coated dressing was dried under vacuum.

The surface of the cotton coated with the HNE commercial substrates was imaged by HRSEM and fluorescence emission after incubation at $37^{\circ} \mathrm{C}$ with several concentrations of HNE. The emission was quantified in a fluorimeter and visually detected by using an UV light lamp.

\section{Characterization of the FRET peptides and chemical immobilization by using GTA crosslinking}

To examine the ability of FRET peptides FAM-AAPV-Dabcyl and EDANS-AAPV-Dabcyl in detecting HNE activity, different peptide concentrations $(1-600 \mu \mathrm{M})$ were added to a fixed amount of HNE solution $(0.5 \mathrm{U} / \mathrm{mL})$. The incubation was carried out at $37{ }^{\circ} \mathrm{C}$, and at determined time points, the fluorescence spectrum $(400-700 \mathrm{~nm})$ of the samples was registered in a fluorimeter. As suggested by the supplier, the peptide was dissolved in DMSO to a stock concentration of $2.7 \mu \mathrm{g} / \mathrm{mL}$ and then diluted to the required assay concentrations in the reaction buffer.

Chemical immobilization of FRET peptides into polyamide dressing consisted in the following steps: (1) surface alkaline treatment of previously washed polyamide dressing in $2 \mathrm{~g} / \mathrm{L}$ $\mathrm{NaOH}$ for $30 \mathrm{~min}$ at room temperature with agitation assisted with glass beads, (2) GTA crosslinking to FRET peptide for $2 \mathrm{~h}$ with vigorous agitation and (3) reaction of treated polyamide dressing $\left(1 \times 1 \mathrm{~cm}^{2}\right)$ with GTA-FRET peptide under orbital agitation overnight at $4{ }^{\circ} \mathrm{C}$ (Fig. 2). At the end of each step, treated dressings were washed and dried under vacuum. The dye pararosaniline (PS) was used as a visual control of the immobilization, and its GTA crosslinking was adapted from a previously described protocol of Monro et al. (2012). Crosslinking with GTA was performed in the molar ratios of 1:125 for FRET peptide and GTA and 1:125:1255 for control PS, $\mathrm{HCl}$ and GTA.

\section{Results}

\section{In vitro characterization of commercially available fluorogenic HNE substrates sonochemically immobilized on wound dressings}

As a fluorogenic substrate, we used MeOSuc-AAPV-AFC. Cleavage of this substrate releases the highly fluorescent compound 7-amino-4-trifluormethylcoumarin (AFC) $\left(\lambda \mathrm{Ex}_{\max }=380 \mathrm{~nm} ; \lambda \mathrm{Em}_{\max }=460 \mathrm{~nm}\right)($ Scharpé et al. 1988). The chromogenic peptide Glp-PV-pNA was used as a negative control of fluorescence. The enzymatic hydrolysis of this substrate releases para-nitroaniline $(p \mathrm{Na})$, a molecule that can be easily detected with a UV-Vis spectrophotometer by increase in absorbance $\left(\lambda \mathrm{Abs}_{\max }\right)$ at $400 \mathrm{~nm}$ which corresponds to the 
Table 1 Chemical information (structure, molecular weight and spectral properties) of the used HNE substrates, Glp-PV- $p$ NA, MeOSuc-AAPV-AFC, FAM-AAPV-Dabcyl and EDANS-AAPV-Dabcyl

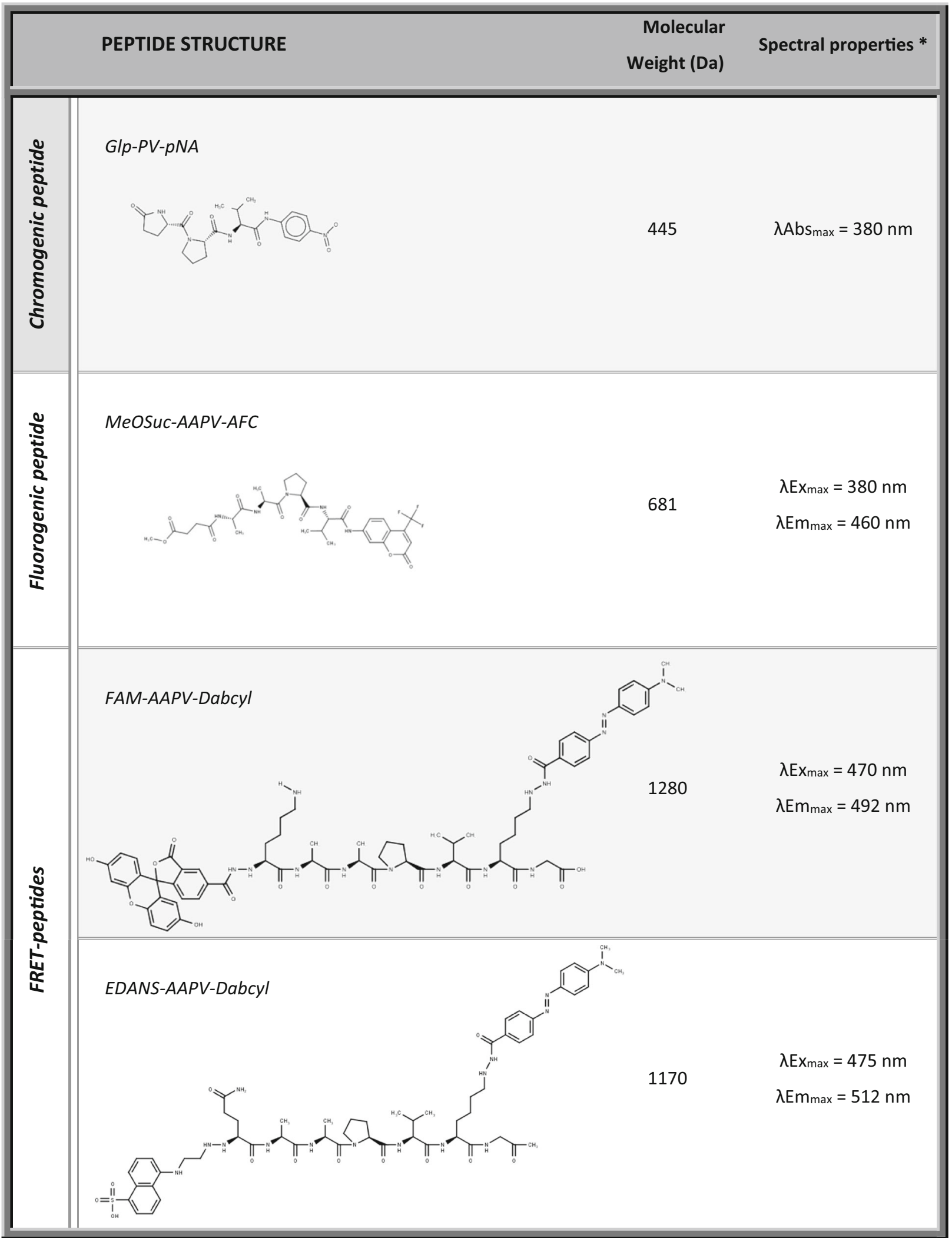

All structures were drawn by using MarvinSketch (ChemAxon, HU)

${ }^{a}$ Determined by using the reaction buffer of $\mathrm{HNE}$ 


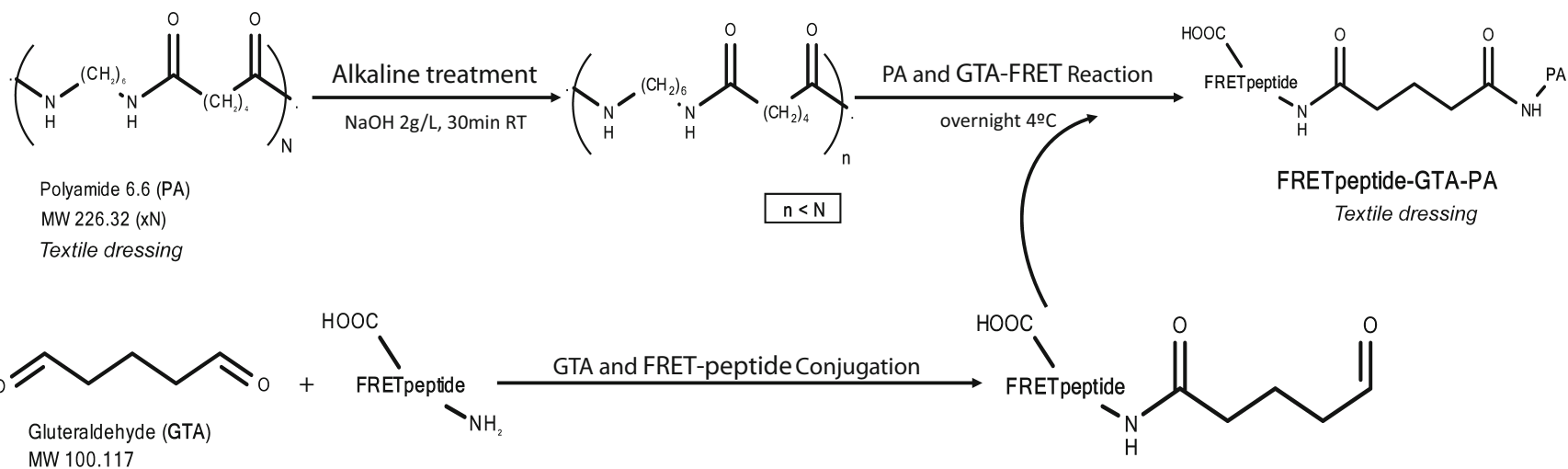

EDANS/Dabcyl

FRET-Peptide

FRETpeptide-GTA

MW 1170.36

Fig. 2 Chemical reaction of the EDANS/Dabcyl FRET peptide immobilization into a polyamide (PA) dressing in three steps: alkaline treatment of the PA dressing surface, conjugation of glutaraldehyde

appearance of a light yellow colour detected by the naked eye (Bru and Walde 1991).

These two commercially available peptides specific for HNE were immobilized into a plain and commonly used cotton gauze. Immobilization was accomplished by a sonochemical method by using an ultrasonic (US) probe immersed in a solution of the substrate where the dressing was placed.

The morphology of the cotton dressing before and after the sonochemical coating reaction was observed by HRSEM (Fig. S1). A dense layer of spherical peptide NPs with an average particle diameter of $\sim 15 \mathrm{~nm}$ was observed on the cotton fibres after sonication (Fig. S1 B-D).

In order to evaluate the availability of the immobilized substrates to the enzyme, the sonochemically treated dressings were incubated with HNE and placed in a dark chamber to be observed under UV light (366 nm) source (Fig. 3).

Successfully, HNE was able to cleave MeOSuc-AAPV$\mathrm{AFC}$, as the dressing turned fluorescent after the addition of the enzyme (Fig. 3a). Glp-PV-pNa was also efficiently immobilized and accessible to the enzyme, as a slightly yellow colour was visible after incubation of the dressing with HNE (data not shown). However, this colour change is barely detected by the human eye. As expected, this substrate did not develop fluorescence upon cleavage by HNE (Fig. 3b).

For an estimate quantification of the naked eye visualizations, the fluorescence spectrum was measured for each dressing (Fig. 4b). The fluorescence intensity increased proportionally according to the concentration of HNE enzyme (Fig. 4).

\section{In vitro characterization of FRET peptides for HNE detection}

Two fluorophore donors, 5(6)-carboxyfluorescein (FAM) or $N$-(aminoethyl)-5-naphthalenamine-1-sulfonic acid (EDANS), and a quencher acceptor, Dabcyl (4-([4'-
(GTA) and FRET peptide, and reaction of the PA-treated dressing and GTA-FRET peptide. All structures were drawn by using MarvinSketch (ChemAxon, HU)

dimethulamino)phenyl]azo)benzoyl), were covalently linked on opposite terminals of the HNE-specific peptide sequence, specifically the donor to the N-terminal and the quencher to the C-terminal. To avoid disturbance in the core of the peptide cleavage sequence, some amino acid residues were added to the sequence to serve as anchors through the free amino groups. Namely, FAM and Dabcyl were coupled through a lysine residue, and EDANS was coupled through a glutamine residue (Table 1). A glycine residue was also added at $\mathrm{C}$ terminal to improve the synthesis and purification process.

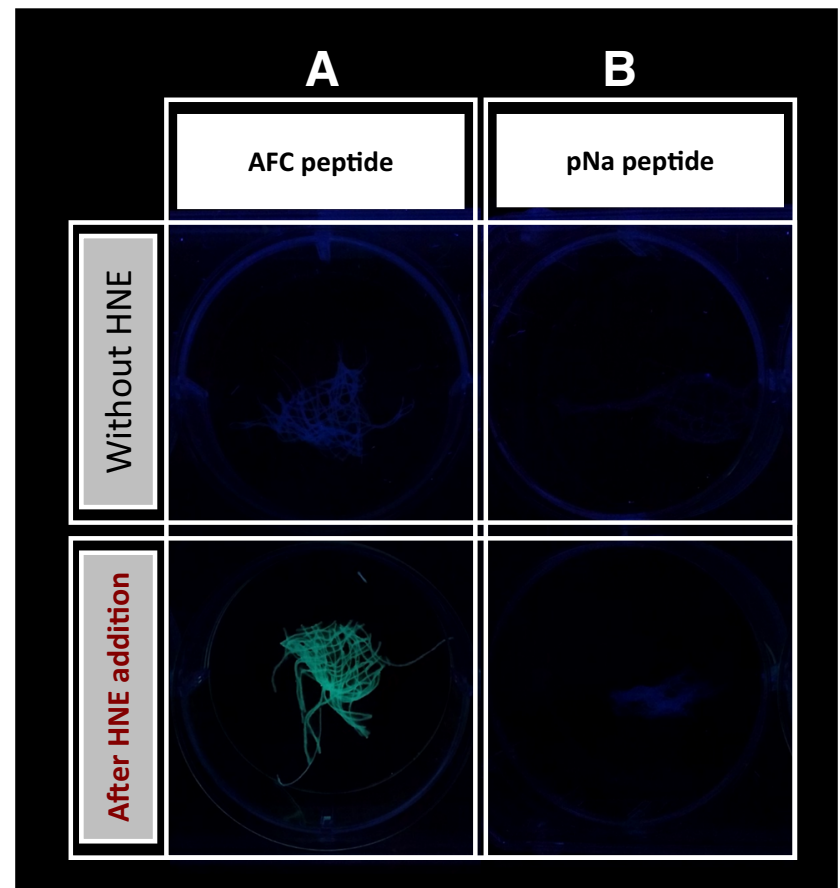

Fig. 3 Visual assessment of the cotton dressing coated with the HNE substrates a MeOSuc-AAPV-AFC or b Glp-PV- $p$ NA, after the addition of reaction buffer without $\mathrm{HNE}$ and with $150 \mathrm{mU}$ of enzyme (incubation at $37^{\circ} \mathrm{C}, 30 \mathrm{~min}$ ) by using UV light with $366 \mathrm{~nm}$ as excitation source 
A

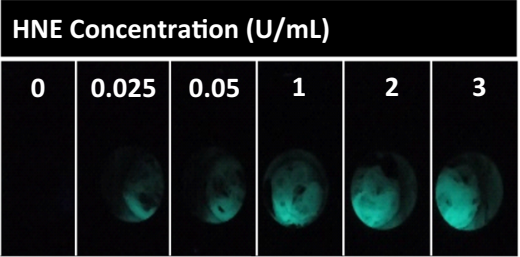

B Effect of Incubation time with HNE

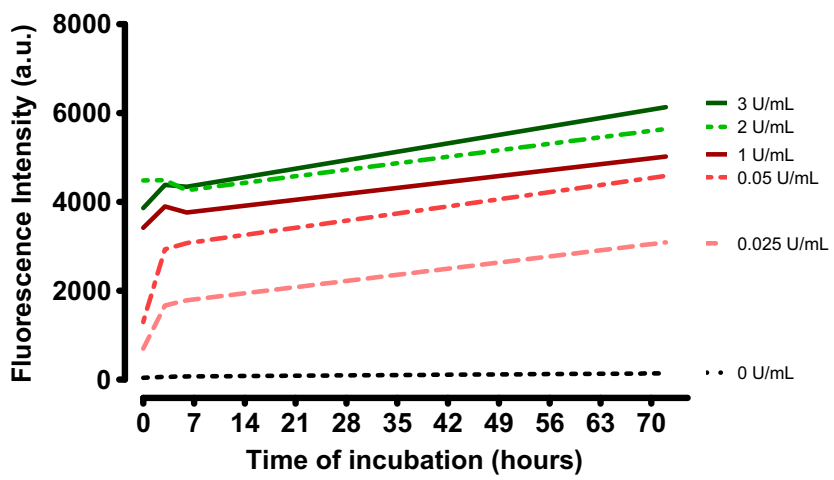

Fig. 4 Assessment of the sensitivity of the substrate MeOSuc-AAPVAFC with different concentrations of $\mathrm{HNE}(0-3 \mathrm{U} / \mathrm{mL})$. a Visual assessment of the fluorescence of the dressing, using UV light (366 nm) as excitation source. b Fluorescence intensity of the dressing material quantified in a fluorimeter with $\lambda E x_{\max }=380 \mathrm{~nm}$ and emission $\lambda \mathrm{Em}_{\max }=460 \mathrm{~nm}$ over time of incubation at $37^{\circ} \mathrm{C}$. The legend shows the categorization of the fluorescence value in arbitrary units (a.u.)

Because there are no previous studies with these substrates for HNE, first we analysed in detail the spectral changes of the donor fluorophore in the absence and presence of the HNE to assess the ability of these FRET peptides to serve as reporter for HNE activity. Several concentrations of each FRET peptide were tested, and the obtained results are presented in the following Fig. 5 and Fig. S2.

For both FRET peptides, the fluorescence intensity of the donor, FAM or EDANS, at the wavelength for the peak of fluorescence emission $\left(\lambda \mathrm{Em}_{\max }\right.$ of 492 and $512 \mathrm{~nm}$, respectively) increased when the enzyme was added (Fig. S2). The fluorescence intensity was also higher with the increase of substrate concentrations (Fig. S2 and Fig. 5). This correlation was expected since the fluorophore donor molecules present in solution increase in number and, therefore, more photons are capable to be released.

Then, the FRET peptide solutions were placed on a hydrophobic paper surface for visual assessment on a solid surface, and the fluorescence changes upon HNE cleavage were visualized by using a UV light lamp (Fig. 6). It was possible to visually observe significant differences in the fluorescence intensity of both FRET peptides in the absence or presence of HNE enzyme; however, the fluorescence changes were significantly higher for EDANS/Dabcyl peptide. For this reason, this FRET peptide pair was selected for further immobilization into a textile dressing.
A FAM-Dabcyl Peptide
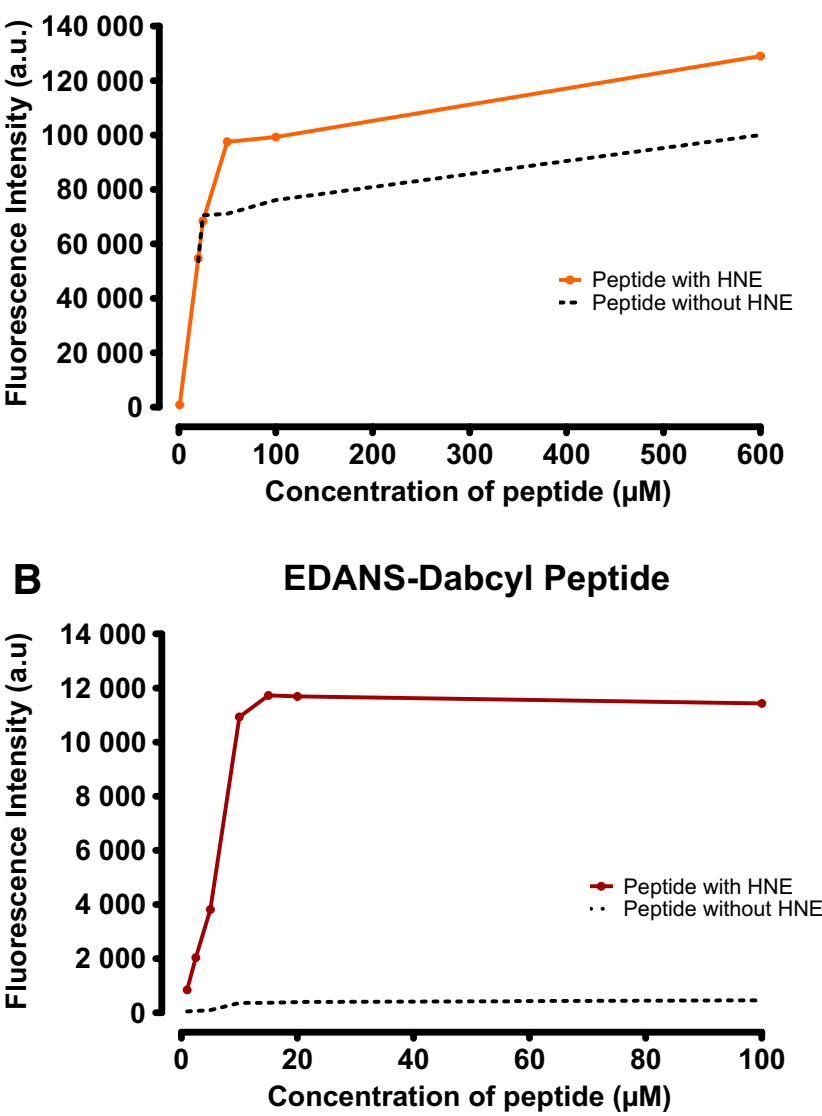

Fig. 5 Fluorescence intensity of a FAM/Dabcyl peptide and b EDANS/ Dabcyl peptide before (dashed line) and after a 10-min incubation at $37{ }^{\circ} \mathrm{C}$ with HNE (10-600 or 1-100 $\mu \mathrm{M}$, respectively) (filled coloured line). $\lambda \mathrm{Em}_{\max }$ was chosen accordingly for each fluorophore donor (Table 1), and the emission spectra were measured. The legend shows the categorization of the fluorescence value in arbitrary units (a.u.)

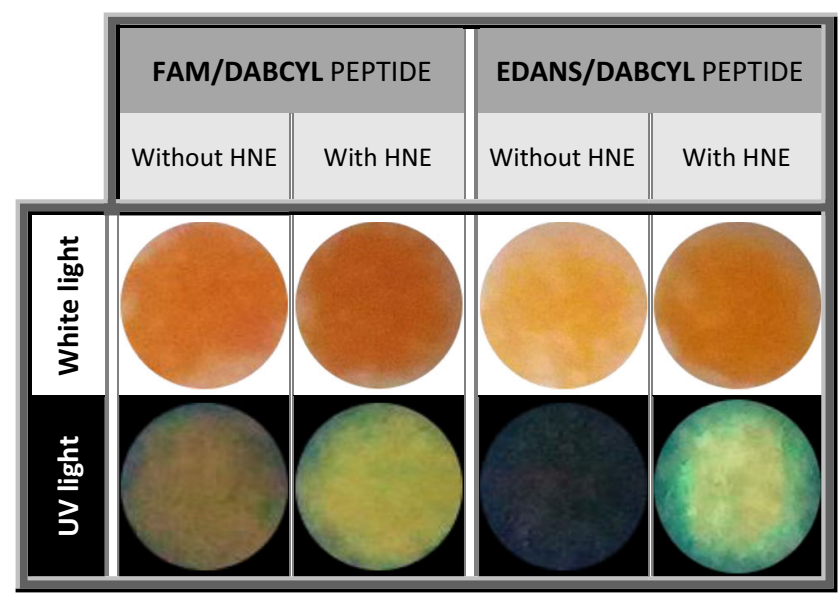

Fig. 6 FRET peptides FAM/Dabcyl and EDANS/Dabcyl $(100 \mu \mathrm{M}$ of concentration) solutions after incubation with $150 \mathrm{mU}$ of $\mathrm{HNE}$ at $37^{\circ} \mathrm{C}$ for $10 \mathrm{~min}$. The photographs show visualization under white light and UV light from a portable UV device. Sample solutions were placed in a hydrophobic paper surface 
In vitro characterization of EDANS/Dabcyl FRET peptide for HNE detection chemically immobilized on wound dressings

The FRET peptide EDANS/Dabcyl was then immobilized into a traditional textile dressing of polyamide based in a methodology described in Silva et al. (2007), as schematized in Fig. 2. Photographs of the resulted PA dressings after immobilization are presented in Fig. 7a. The immobilization efficiencies, calculated as the fraction of the amount of peptide that remained in the dressing after washing compared to the initially immobilized/adsorbed amount, are presented in Fig. 7b. Statistical significance of the immobilization efficiencies was determined by Student's $t$ test with a confidence level of $95 \%$ and is assigned in the graph.

The immobilization efficiency for $\mathrm{PS}-\mathrm{HCl}$ dye (used in this work as a control) varied greatly whether or not GTA was used to crosslink it to PA. GTA crosslinking method allowed a significant increase in the immobilization efficiency ( $P$ value $<0.0001)$, changing from $38.32 \pm 1.04$ to $69.81 \pm 0.46 \%$.

The solution of EDANS/Dabcyl FRET peptide has an orange colour (Fig. 6); however, after the immobilization process, its colour intensity faints to a pale yellow (Fig. 7a). EDANS/Dabcyl FRET peptide was successfully immobilized into the polyamide dressing with similar immobilization efficiency of $77.94 \pm 2.45 \%$ with GTA and $79.62 \pm 2.38 \%$ without GTA (Fig. 7b) (ns nonsignificant, $P$ value of 0.44 ).

For the final application of using the treated fabric as a sensor for elastase activity, the dressings functionalized with EDANS/Dabcyl FRET peptide were incubated with the HNE enzyme (Fig. 7c). A slight fluorescence signal was detected upon HNE addition after $24 \mathrm{~h}$ of incubation. However, the fluorescence intensity was lower when the substrate was immobilized than when in solution (Fig. 6). It also required an increased incubation time with elastase (24 $\mathrm{h}$ of incubation at $37^{\circ} \mathrm{C}$ ) in comparison to the previous assays in solution (10 $\mathrm{min})$.
Fig. 7 a Visual assessment under white light of the resulted PA dressings after immobilization without GTA and with GTA of control PS-HCl dye and EDANS/ Dabcyl. b Immobilization efficiencies of PS-HCl dye and EDANS/Dabcyl FRET peptide with and without GTA crosslinking. c Visual assessment under UV light of the EDANS/ Dabcyl FRET peptide immobilized on PA dressings with GTA crosslinking method upon incubation with $150 \mathrm{mU}$ of $\mathrm{HNE}$ at $37^{\circ} \mathrm{C}$ for $24 \mathrm{~h}$

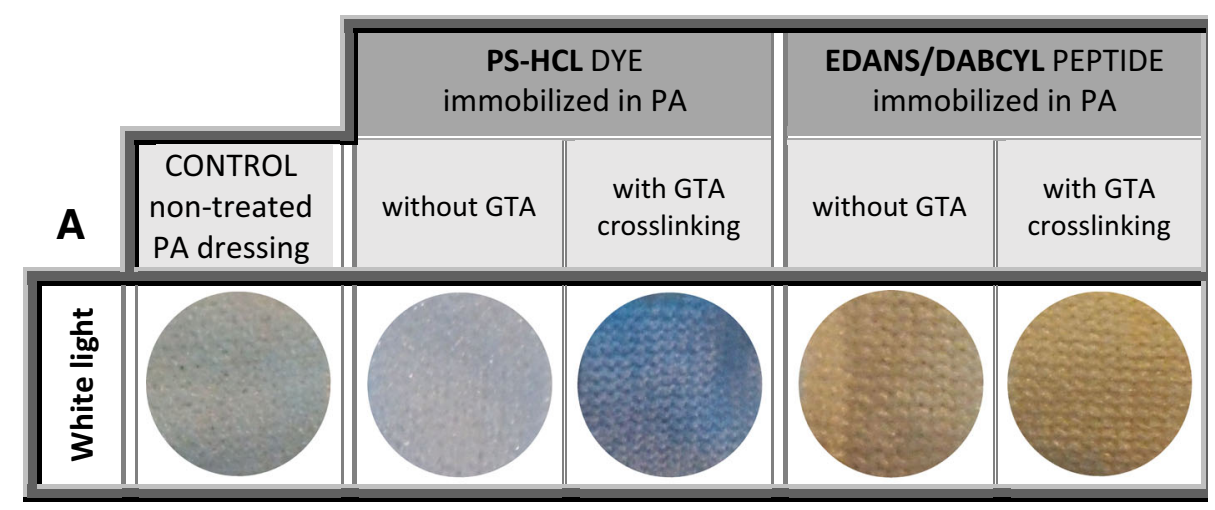

Method without GTA

B Z Method with GTA crosslinking

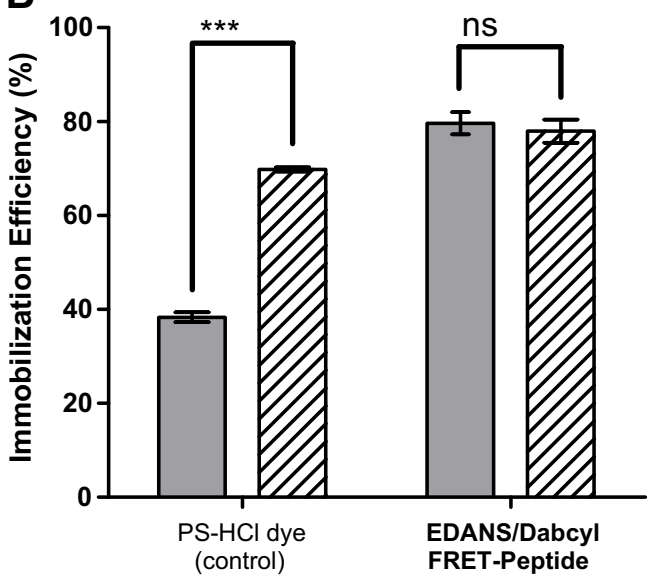




\section{Discussion}

\section{Analysis of the developed fluorogenic sensors for HNE detection}

In this work, we intend to immobilize fluorogenic substrates on wound dressings to efficiently detect HNE present in exudate from chronic wounds.

As a first and straightforward immobilization strategy, we submitted cotton dressings and substrate solutions to an ultrasound treatment leading to the formation of peptide NPs which are thrown at the surface of the dressing (Fig. S1 B-D). The formation of these peptide NPs only occurred as a result of ultrasound irradiation applied on the peptide solution with the dressing, since before sonication, the cotton fibres presented a smooth structure (Fig. S1 A). This result is in accordance with previous publications that report on the sonication of organic solutions leading to the formation of NPs (Kiel et al. 2012; Perelshtein et al. 2014). Nevertheless, these do not remain stable in aqueous solution without a stabilizer and eventually re-dissolve back in the solvent. If one wants to embed organic NPs onto a solid surface, then the use of a sonochemical technique presents itself as a clear benefit, since in a one-step process, the organic peptide NPs are created from the aqueous solution and anchored onto the cotton surface.

Detection of HNE activity with the fluorogenic MeOSuc-AAPV-AFC substrate showed to be very sensitive, since visual detection by using a UV light was possible even at low concentrations of the enzyme (Fig. 4). Even so, a second approach by using peptides with specific cleavage sequence for HNE that were coupled to a fluorescence quenching system was also studied. According to the literature, the use of FRET system allows a more sensitive detection of the peptide cleavage in comparison to colorimetric (and even other fluorescence) methods (Lakowicz 2006; Adhikari et al. 2014).

In this work, two FRET peptide pairs FAM/Dabcyl and EDANS-Dabcyl were tested. Upon HNE addition, the fluorescence of both FRET peptides increased (Fig. S2). This means that HNE was able to recognize the specific cleavage sequence, and the subsequent peptidolysis on the FRET peptides separated the fluorophore donor and the quencher acceptor molecules. Consequently, Dabcyl was no longer in the necessary spatial vicinity of the donors to quench its fluorescence, and hence, their signal is released to the medium. Dabcyl is a dark quencher, which means that instead of emitting the energy that was transferred from the donor, it releases it as heat (Lakowicz 2006). Since the energy emission of the acceptor molecule will not affect the fluorescence measurements, the use of dark quencher enables a more sensible analysis (Lakowicz 2006), usually from an off-signal to an on-signal.
Analysing in detail, significant differences are seen between the fluorescence profiles of FAM/Dabcyl and EDANS/Dabcyl pairs before and after HNE cleavage (Fig. 5). The basal fluorescence (i.e. without HNE) of FAM/ Dabcyl pair is much higher than that of EDANS/Dabcyl pair. This then translates on a fluorescence change after HNE addition more evident when using EDANS than FAM as a donor, even with the relative fluorescence intensity of cleaved EDANS/Dabcyl peptide being 10 times lower than cleaved FAM/Dabcyl peptide. The main reason for these results resides in the spectral overlap between the donor and acceptor (Fig. S3).

Resonance energy transfer only occurs when the acceptor molecule is able to absorb light in the same spectral range as the donor emission, meaning that the energy of photon emitted by the donor should be as close as possible to the energy absorption ability of the acceptor (Medintz and Hildebrandt 2013). In practical terms, this means that the emission spectrum of the donor should overlap the absorption spectrum of the acceptor, being this termed as donor/acceptor pair compatibility (Szöllosi et al. 1998; Tabassum et al. 2012; Medintz and Hildebrandt 2013; Masters 2014).

As presented in Fig. S3 A, for FAM/Dabcyl, there is no complete spectral overlap and FAM emission is not entirely absorbed by the quencher Dabcyl. Since the donor fluorescence quenching only occurs at the "overlapping area", a great amount of fluorescence emission leaks even when the peptide is not cleaved, and for this reason, the fluorescence changes without or with HNE are not significant. These results demonstrated the non-compatibility of the FRET pair FAM/ Dabcyl despite being commercially available.

For the other FRET pair, EDANS emission is almost fully absorbed by Dabcyl, as showed by the complete spectral overlap (Fig. S3 B). The basal fluorescence of EDANS/Dabcyl peptide is very weak; thus, upon peptide cleavage, the increase in fluorescence is notable. EDANS/Dabcyl peptide proved to be very sensitive to HNE activity, as the concentrations tested with HNE were much lower than for the other FRET peptide (Fig. 5).

The fluorescence visual changes were also significantly higher for EDANS/Dabcyl peptide (Fig. 6), since the condition without HNE was completely visually off, and after the enzyme addition, a bright signal appeared. FAM/Dabcyl peptide showed to be fluorescent even in the absence of HNE, which for our purposes would lead to false positive results of HNE assessment in wound fluids. The evaluation of HNE activity on the dressing must rely on UV excitation and fluorescence visualization, since observation of the dressings under white light revealed no major differences in colour generation after HNE addition.

To enhance and create a stronger embedding, a chemical immobilization method was favoured as an alternative to previously used method of sonochemical immobilization by US 
probe. The chemical immobilization chosen consisted in the establishment of chemical bonds between the amine groups of the peptide (N-terminal) and free amines from polyamide generated by mild hydrolysis through a GTA crosslinking conjugation (Fig. 2). GTA is one of the most effective protein crosslinking reagents due to its high reactivity and low cost (Migneault et al. 2004). Since GTA is extremely reactive to amine groups, a polyamide textile dressing was preferred instead of the previously used cotton bandage. Furthermore, polyamide dressing provides high elasticity and strength, textile uniformity and cost effectiveness, presenting itself as a great immobilization substrate for wound dressing applications (Herrick et al. 1992b).

Pararosaniline (PS) dye was used as a control of the immobilization process since it is described as a reactive dye of polymer fibres with high tinting properties, i.e. high immobilization efficiencies (Bowers 2000). In addition, in our experiment, PS also allowed a visual confirmation of the chemical reactions during the immobilization process, since PS is used as main reagent in colorimetric tests for aldehydes (Bowers 2000; Monro et al. 2012). In aqueous neutral solution, PS colour is bright magenta, but upon acidification with $\mathrm{HCl}$, it turns to a pale biscuit colour; after the addition of aldehyde compounds, PS solution results in a purple blue colour (Monro et al. 2012). Figure 7a confirms these colour changes of PA dressing immobilized with $\mathrm{PS}-\mathrm{HCl}$ in a direct reaction (control without GTA) and with GTA crosslinking.

A high immobilization efficiency of FRET peptide without GTA crosslinking was not expected (Fig. 7b); in fact, GTA was initially selected and used to enhance the immobilization process. According to the findings available in literature, this surprising result might be owed to the initial alkaline treatment of the polyamide dressing. Conventionally, alkaline or acid solvent hydrolysis is used to improve the hydrophilicity and dyeability of textiles (Gashti et al. 2011; Begum et al. 2016). By using the initial alkaline treatment with $\mathrm{NaOH}$, the polyamide structure suffered a hydrolysis process on the most outward fibres that improved its absorption properties, allowing an enhanced molecule penetration (Gashti et al. 2011; Begum et al. 2016), in this case of the EDANS/Dabcyl peptide. On the contrary, immobilization efficiencies with non-treated PA dressings are described to be considerably lower (Silva et al. 2007; Gashti et al. 2011; Begum et al. 2016).

Upon HNE cleavage, a slight fluorescence signal was detected (Fig. 7c); however, its intensity was lower in comparison to the observed signal in solution (Fig. 6). This decreased detection might be related to the inaccessibility of the immobilized FRET peptide. Indeed, substrate immobilization significantly changes enzymatic reaction parameters, in which the rates and yields of solid-phase reactions are often lower than solution-phase reactions (Laurent et al. 2008; Gray et al. 2013). Since HNE active site possesses a hemispherical and hydrophobic deep pocket which is completely buried in the interior of the enzyme, it is likely that HNE is less capable to act on a substrate which is immobilized on a solid surface like our EDANS/Dabcyl FRET peptide chemically conjugated to the PA dressing (Korkmaz et al. 2008b; Gray et al. 2013).

Furthermore, the intermolecular quenching phenomenon from Dabcyl on EDANS cannot be excluded as a possible reason for the observed diminished detection (Korkmaz et al. 2008a). In solution, we recorded that upon HNE cleavage, the full fluorescence quantum yield of EDANS was restored (Fig. 6), since the now released Dabcyl peptide fragment out distanced from EDANS. However, this distance detachment may be affected in the solid state. When immobilized, the distance of the cleaved fragments of EDANS and Dabcyl peptide might not significantly increase, since EDANS might be still close to its previously paired Dabcyl or to Dabcyl from molecules in the vicinity (Korkmaz et al. 2008a). This phenomenon termed as intermolecular quenching could also contribute to the weaker fluorescence observed in Fig. 7c.

\section{Improvements and future prospects}

Although the obtained fluorescent on-signal of EDANS/ Dabcyl functionalized dressing was at this stage limited, this is a promising approach for specific detection of elastase on sensor dressings by using a UV portable light. Currently, optimization of the immobilization process of the FRET peptide onto a textile dressing is necessary. The chosen chemical immobilization method with GTA crosslinking caused some problems and restricted the accessibility of the enzyme to the detection substrate. A popular method that can be applied to improve these issues is the use of polyethylene glycol (PEG) spacers that would increase the substrate accessibility and mobility. As a result, the HNE enzyme could access and catalyse the immobilized FRET peptide, causing an intense fluorescent on-signal (Laurent et al. 2008; Gray et al. 2013).

In further development of this work, the immobilization of the HNE-specific FRET peptide onto the dressing must be optimized taking into consideration not only the method to be applied but also the conditions and parameters used, including the concentration of the FRET peptide. At this stage, the in vitro assays were only performed by using $150 \mathrm{mU}$ of commercial HNE enzyme (specifically, $50 \mu \mathrm{L}$ with $3 \mathrm{U} / \mathrm{mL}$ ). Since HNE activity in non-infected or infected wound ranges from $2.89 \pm 1.27$ to $22.97 \pm 13.27 \mathrm{U} / \mathrm{mL}$ (Hasmann et al. 2011), it is paramount that the HNE sensor develops a fluorescent signal proportional to the activity of HNE in the wound. For that, in further assays, the concentration of immobilized FRET peptide in the dressing should be optimized by using exudate collected from patients with noninfected and infected wounds, taking into account not only the activity and kinetics of HNE in the exudate but also the 
volume of exudate produced by the wound (high or low exudative wounds).

The $\mathrm{pH}$ and temperature of a wound are dynamic factors that fluctuate depending on the type of wound, local wound factors and systemic factors intrinsic to the patient conditions (Schneider et al. 2007). Under normal circumstances, the $\mathrm{pH}$ value of the skin surface varies between 4 and 6 . However, in a wound, this skin's acidic milieu is disturbed and can oscillate to $\mathrm{pH}$ values above 7-9 (Schneider et al. 2007). Additionally, the temperature of a wound varies from 30 to $42{ }^{\circ} \mathrm{C}$ (Dini et al. 2015). At this stage, evaluation of the developed fluorogenic sensors was performed at the enzyme optimal conditions ( $\mathrm{pH} 7.5$ and temperature $37{ }^{\circ} \mathrm{C}$ ) (Owen and Campbell 1999). To ensure the effectiveness of the HNE sensor and taking into consideration the wound environment, further assays should be performed at wider range of $\mathrm{pH}(4-9)$ and temperature $\left(30-42{ }^{\circ} \mathrm{C}\right)$.

The embedding of chromogenic or naked eye detection sensors into a wound dressing material will allow in situ and real-time assessment of the wound inflammation/infection at an early stage. In contrast to other off-site methods, this novel approach would provide a fast and objective diagnosis, without the need of expensive and long-lasting analytical methods (Mustoe et al. 2006; Gottrup et al. 2010; Harding et al. 2011). The use of a FRET peptide with a HNE-specific cleavage applied in a wound dressing can assist the medical staff in the evaluation of the wound status by fast and easy visualization of the dressing with a portable UV light source (Fig. 1).

Some concerns may be raised regarding the toxicity of fluorogenic substrates used in the sensor, namely the AFC, FAM, EDANS and Dabcyl compounds. Previously, published works have tested these compounds in cell-based assays, where it has been proven their safety. Actually, AFC has been extensively used as a viable cell protease marker due its innocuous behaviour on cells (Niles et al. 2007; Niles et al. 2009). Also, previous studies have shown viability rates above $98 \%$ of intact THP- 1 cells incubated with $5 \mu \mathrm{M}$ of FAM peptide substrates (Alvarez-Iglesias et al. 2005), as well as concentrations up to $100 \mu \mathrm{M}$ of EDANS/Dabcyl compounds have shown no cytotoxicity effect on the viability of human rhabdomyosarcoma (RD) cells (Shang et al. 2015).

Although a proven absence of toxicity can only be evaluated with direct tests to evaluate the effect of these fluorogenic sensors on cells, we may predict that they should be biocompatible and will not lead to cytotoxicity due to the incorporated fluorogenic compounds. Furthermore, at the next stages of this work, the fluorogenic sensors will be placed on top of a dressing with controlled fluid handling ability, so that the wound fluid will only flow in the direction of the upper surface. This way, the released products upon HNE cleavage would not leak to the wound. The immobilization site containing the fluorogenic sensor will also be protected against light and air exposure with a removable dark cover, allowing the use of the same dressing for longer periods of time consistent with the typical wound care practice, without the risk of fluorescence bleaching.

Chronic wounds are a significant burden for the health care system, accounting for almost $4 \%$ of its total budget, and currently increasing (Mustoe et al. 2006; Sen et al. 2010). Early detection of incipient infection in chronic wound reduces the severity of the disease and decreases health care expenses (Macgregor et al. 2008). However, currently, it is very difficult, expensive and time consuming to accurately assess a wound status (Moore et al. 2006; Sen et al. 2010). Besides, upon diagnosis, the current treatment strategies follow a trial-and-error approach, which is also inefficient, expensive and time-consuming (Moore et al. 2006; Gottrup et al. 2010).

This work serves as a proof of concept, so to this extend, it aims only to demonstrate the feasibility of a fluorogenic sensor for monitoring the activity of HNE and not to be an early version of a final product. We envisioned that this new functional and diagnostic material will allow a better control of inflammation/infection in chronic wound at an early stage, contributing to the decision on the treatment to be implemented and, consequently, to a better patient's life quality and decrease in the wound care costs.

Acknowledgments We would like to acknowledge the European project InFact-Functional materials for fast diagnosis of wound infection (FP7-NMP-2013-SME-7-grant agreement no. 604278) for funding the work and also the grant for Joana Cunha. Ana Ferreira would also like to acknowledge the Portuguese Foundation for Science and Technology (FCT) for the $\mathrm{PhD}$ student grant SFRH/BD/113247/2015. The work done at Centre of Biological Engineering (CEB) was supported by FCT under the scope of the strategic funding of UID/BIO/04469/2013 unit, COMPETE 2020 (POCI-01-0145-FEDER-006684) and BioTecNorte operation (NORTE-01-0145-FEDER-000004) funded by European Regional Development Fund under the scope of Norte 2020Programa Operacional Regional do Norte.

\section{Compliance with Ethical Standards}

Funding This study was funded by the European project InFactFunctional materials for fast diagnosis of wound infection (FP7-NMP2013-SME-7 — grant agreement no. 604278). The work done at Centre of Biological Engineering (CEB) was also supported by the Portuguese Foundation for Science and Technology (FCT) under the scope of the strategic funding of UID/BIO/04469/2013 unit, COMPETE 2020 (POCI01-0145-FEDER-006684) and BioTecNorte operation (NORTE-010145-FEDER-000004) funded by European Regional Development Fund under the scope of Norte 2020-Programa Operacional Regional do Norte.

Conflict of interest The authors declare that they have no conflict of interest.

Ethical approval This article does not contain any studies with human participants or animals performed by any of the authors. 


\section{References}

Adhikari S, Ghosh A, Mandal S, Sengupta A, Chattopadhyay A, Sanmartín Matalobos J, Lohar S, Das D (2014) Visible light excitable $\mathrm{ON}$ fluorescence and naked eye detection of $\mathrm{Cu}^{2+}$ via hydrolysis of rhodamine-thiophene conjugate: human breast cancer cell (MCF7) imaging studies. Dalton Trans 43:7747-7751. doi: $10.1039 / \mathrm{c} 4 \mathrm{dt} 00002 \mathrm{a}$

Alvarez-Iglesias M, Wayne G, O’Dea KP, Amour A, Takata M (2005) Continuous real-time measurement of tumor necrosis factor- $\alpha$ converting enzyme activity on live cells. Lab Investig 85:1440 1448. doi:10.1038/labinvest. 3700340

Barros SC, Louro RO, Micaêlo NM, Martins JA, Marcos JC, CavacoPaulo A (2013) NMR and molecular modelling studies on elastase inhibitor-peptides for wound management. React Funct Polym 73: 1357-1365. doi:10.1016/j.reactfunctpolym.2013.02.011

Begum S, Wu J, Takawira CM, Wang J (2016) Surface modification of polyamide 6, 6 fabrics with an alkaline protease-Subtilisin. J Eng Fiber Fabr 11:64-74

Bowers JS (2000) Toluidines. In: Ullmann's encyclopedia of industrial chemistry. Wiley-VCH Verlag GmbH \& Co. KGaA, Weinheim, pp. 119-126

Brena B, González-Pombo P, Batista-Viera F (2013) Immobilization of enzymes: a literature survey. In: Immobilization of enzymes and cells: third edition. pp 15-31

Bru R, Walde P (1991) Product inhibition of alpha-chymotrypsin in reverse micelles. Eur J Biochem 199:95-103

Carmona AK, Juliano MA, Juliano L (2009) The use of fluorescence resonance energy transfer (FRET) peptides for measurement of clinically important proteolytic enzymes. An da Acad Bras Ciências 81: 381-392

Dini V, Salvo P, Janowska A, Di Francesco F, Barbini A, Romanelli M (2015) Correlation between wound temperature obtained with an infrared camera and clinical wound bed score in venous leg ulcers. Wounds a Compend Clin Res Pract 27:274-278

Fan H, Jiang X, Zhang T, Jin Q (2012) Peptide-induced fluorescence quenching of conjugated polyelectrolyte for label-free, ultrasensitive and selective assay of protease activity. Biosens Bioelectron 34: 221-226. doi:10.1016/j.bios.2012.02.006

Gashti MP, Willoughby J, Agrawal P (2011) Surface and bulk modification of synthetic textiles to improve dyeability. Text Dye 18:927929. doi: $10.5772 / 800$

Gedanken A (2004) Using sonochemistry for the fabrication of nanomaterials. Ultrason Sonochem 11:47-55. doi:10.1016/j. ultsonch.2004.01.037

Gottrup F, Apelqvist J, Price P (eds) (2010) Outcomes in controlled and comparative studies on non-healing wounds: recommendations to improve the quality of evidence in wound management. J Wound Care 19:237-268. doi:10.12968/jowc.2010.19.6.48471

Gray CJ, Weissenborn MJ, Eyers CE, Flitsch SL (2013) Enzymatic reactions on immobilised substrates. Chem Soc Rev 42:6378. doi: $10.1039 / \mathrm{c} 3 \operatorname{cs} 60018 \mathrm{a}$

Harding K, Armstrong DG, Barrett S, Kaufman H, Lázaro-Martínez JL, Mayer D, Moore Z, Romanelli M, Queen D, Schultz G, Serena T, Sibbald G, Snyder R, Strohal R, Vowden K, Vowden P, Paolo Zamboni (2011) International consensus. The role of proteases in wound diagnostics. An expert working group review

Hasmann A, Gewessler U, Hulla E, Schneider KP, Binder B, Francesko A, Tzanov T, Schintler M, Van der Palen J, Guebitz GM, Wehrschuetz-Sigl E (2011) Sensor materials for the detection of human neutrophil elastase and cathepsin G activity in wound fluid. Exp Dermatol 20:508-513. doi:10.1111/j.1600-0625.2011.01256.x

Herrick SE, Sloan P, McGurk M, Freak L, McCollum CN, Ferguson MW (1992a) Sequential changes in histologic pattern and extracellular matrix deposition during the healing of chronic venous ulcers. Am J Pathol 141:1085-1095

Herrick SE, Sloan P, McGurk M, Freak L, McCollum CN, Ferguson MW (1992b) Sequential changes in histologic pattern and extracellular matrix deposition during the healing of chronic venous ulcers. Am J Pathol 141:1085-1095

Kiel S, Grinberg O, Perkas N, Charmet J, Kepner H, Gedanken A (2012) Forming nanoparticles of water-soluble ionic molecules and embedding them into polymer and glass substrates. Beilstein $\mathrm{J}$ Nanotechnol 3:267-276. doi:10.3762/bjnano.3.30

Kolaczkowska E, Kubes P (2013) Neutrophil recruitment and function in health and inflammation. Nat Rev Immunol 13:159-175. doi:10.1038/nri3399

Korkmaz B, Attucci S, Juliano MA, Kalupov T, Jourdan M-L, Juliano L, Gauthier F (2008a) Measuring elastase, proteinase 3 and cathepsin $\mathrm{G}$ activities at the surface of human neutrophils with fluorescence resonance energy transfer substrates. Nat Protoc 3:991-1000. doi:10.1038/nprot.2008.63

Korkmaz B, Moreau T, Gauthier F (2008b) Neutrophil elastase, proteinase 3 and cathepsin G: physicochemical properties, activity and physiopathological functions. Biochimie 90:227-242. doi:10.1016 /j.biochi.2007.10.009

Lakowicz JR (2006) Principles of fluorescence spectroscopy. Springer US, Boston

Laurent N, Haddoub R, Flitsch SL (2008) Enzyme catalysis on solid surfaces. Trends Biotechnol 26:328-337. doi:10.1016/j. tibtech.2008.03.003

Macgregor L, Calne S, Day K, Jones J, Pugh A (2008) Wound infection in clinical practice. Int Wound J 5:iii-i11. doi:10.1111/j.1742-481 X.2008.00488.x

Masters BR (2014) Paths to Förster's resonance energy transfer (FRET) theory. Eur Phys J H 39:87-139. doi:10.1140/epjh/e2013-40007-9

Medintz I, Hildebrandt N (2013) FRET-Förster Resonance Energy Transfer. Wiley-VCH Verlag GmbH \& Co. KGaA, Weinheim

Mersmann A (2001) Crystallization technology handbook, a. CRC Press, Mersman

Migneault I, Dartiguenave C, Bertrand MJ, Waldron KC (2004) Glutaraldehyde: behavior in aqueous solution, reaction with proteins, and application to enzyme crosslinking. BioTechniques 37(790-6):798-802. doi:10.2144/3705A0790

Monro TM, Moore RL, Nguyen MC, Ebendorff-Heidepriem H, Skouroumounis GK, Elsey GM, Taylor DK (2012) Sensing free sulfur dioxide in wine. Sensors (Switzerland) 12:10759-10773. doi: $10.3390 / \mathrm{s} 120810759$

Moore K, McCallion R, Searle RJ, Stacey MC, Harding KG (2006) Prediction and monitoring the therapeutic response of chronic dermal wounds. Int Wound J 3:89-98. doi:10.1111/j.17424801.2006.00212.x

Mustoe TA, O’Shaughnessy K, Kloeters O (2006) Chronic wound pathogenesis and current treatment strategies: a unifying hypothesis. Plast Reconstr Surg 117:35S-41S. doi:10.1097/01. prs.0000225431.63010.1b

Niles AL, Moravec RA, Eric Hesselberth P, Scurria MA, Daily WJ, Riss TL (2007) A homogeneous assay to measure live and dead cells in the same sample by detecting different protease markers. Anal Biochem 366:197-206. doi:10.1016/j.ab.2007.04.007

Niles AL, Moravec RA, Riss TL (2009) In vitro viability and cytotoxicity testing and same-well multi-parametric combinations for high throughput screening. Curr Chem Genomics 3:33-41. doi:10.2174 /1875397300903010033

Owen CA, Campbell EJ (1999) The cell biology of leukocyte-mediated proteolysis. J Leukoc Biol 65:137-150

Perelshtein I, Applerot G, Perkas N, Wehrschetz-Sigl E, Hasmann A, Guebitz GM, Gedanken A (2009) Antibacterial properties of an in situ generated and simultaneously deposited nanocrystalline $\mathrm{ZnO}$ on 
fabrics. ACS Appl Mater Interfaces 1:361-366. doi:10.1021 am8000743

Perelshtein I, Ruderman E, Francesko A, Fernandes MM, Tzanov T, Gedanken A (2014) Tannic acid NPs - synthesis and immobilization onto a solid surface in a one-step process and their antibacterial and anti-inflammatory properties. Ultrason Sonochem 21:19161920. doi:10.1016/j.ultsonch.2013.11.022

Perelshtein I, Lipovsky A, Perkas N, Tzanov T, Arguirova M, Leseva M, Gedanken A (2015) Making the hospital a safer place by sonochemical coating of all its textiles with antibacterial nanoparticles. Ultrason Sonochem 25:82-88. doi:10.1016/j. ultsonch.2014.12.012

Perkas N, Amirian G, Dubinsky S, Gazit S, Gedanken A (2007) Ultrasound-assisted coating of nylon 6,6 with silver nanoparticles and its antibacterial activity. J Appl Polym Sci 104:1423-1430. doi:10.1002/app. 24728

Pol VG, Gedanken A, Calderon-Moreno J, Palchik V, Slifkin MA, Weiss AM (2003) Deposition of gold nanoparticles on silica spheres: a sonochemical approach. Chem Mater 15:3402-3402. doi:10.1021 $/ \mathrm{cm} 033005 \mathrm{e}$

Scharpé S, De Meester I, Vanhoof G, Hendriks D, van Sande M, Van Camp K, Yaron A (1988) Assay of dipeptidyl peptidase IV in serum by fluorometry of 4-methoxy-2-naphthylamine. Clin Chem 34: 2299-2301

Schneider LA, Korber A, Grabbe S, Dissemond J (2007) Influence of pH on wound-healing: a new perspective for wound-therapy? Arch Dermatol Res 298:413-420. doi:10.1007/s00403-006-0713-x

Sen CK, Gordillo GM, Roy S, Kirsner R, Lambert L, Hunt TK, Gottrup F, Gurtner GC, Longaker MT (2010) Human skin wounds: a major and snowballing threat to public health and the economy. Wound Repair Regen 17:763-771. doi:10.1111/j.1524-475X.2009.00543.x

Shang L, Zhang S, Yang X, Sun J, Li L, Cui Z, He Q, Guo Y, Sun Y, Yin Z (2015) Biochemical characterization of recombinant enterovirus 71 $3 \mathrm{C}$ protease with fluorogenic model peptide substrates and development of a biochemical assay. Antimicrob Agents Chemother 59: 1827-1836. doi:10.1128/AAC.04698-14
Silva C, Silva CJ, Zille A, Guebitz GM, Cavaco-Paulo A (2007) Laccase immobilization on enzymatically functionalized polyamide $6,6 \mathrm{fi}$ bres. Enzym Microb Technol 41:867-875. doi:10.1016/j. enzmictec.2007.07.010

Sunartio D, Yasui K, Tuziuti T, Kozuka T, Iida Y, Ashokkumar M, Grieser F (2007) Correlation between $\mathrm{Na}^{*}$ emission and "chemically active" acoustic cavitation bubbles. ChemPhysChem 8:2331-2335. doi:10.1002/cphc.200700426

Szöllosi J, Damjanovich S, Mátyus L (1998) Application of fluorescence resonance energy transfer in the clinical laboratory: routine and research. Cytometry 34:159-179. doi:10.1002/(SICI)1097-0320 (19980815)34:4<159::AID-CYTO1>3.0.CO;2-B

Tabassum S, Al-Asbahy WM, Afzal M, Arjmand F, Hasan Khan R (2012) Interaction and photo-induced cleavage studies of a copper based chemotherapeutic drug with human serum albumin: spectroscopic and molecular docking study. Mol BioSyst 8:2424. doi: $10.1039 / \mathrm{c} 2 \mathrm{mb} 25119 \mathrm{a}$

Trevan MD (1988) Enzyme immobilization by covalent bonding. In: New Protein Techniques. Humana Press, New Jersey, pp. 495-510

Vasconcelos A, Pêgo AP, Henriques L, Lamghari M, Cavaco-Paulo A (2010) Protein matrices for improved wound healing: elastase inhibition by a synthetic peptide model. Biomacromolecules 11:22132220. doi:10.1021/bm100537b

Vasconcelos A, Azoia NG, Carvalho AC, Gomes AC, Güebitz G, Cavaco-Paulo A (2011) Tailoring elastase inhibition with synthetic peptides. Eur J Pharmacol 666:53-60. doi:10.1016/j. ejphar.2011.05.056

Weiss SJ, Curnutte JT, Regiani S (1986) Neutrophil-mediated solubilization of the subendothelial matrix: oxidative and nonoxidative mechanisms of proteolysis used by normal and chronic granulomatous disease phagocytes. J Immunol 136:636-641

Weitz JI, Landman SL, Crowley KA, Birken S, Morgan FJ (1986) Development of an assay for in vivo human neutrophil elastase activity. Increased elastase activity in patients with alpha 1proteinase inhibitor deficiency. J Clin Invest 78:155-162. doi:10.1172/JCI112545 